\title{
Multipurpose Monte Carlo simulator for photon transport in turbid media
}

\author{
Pedro Guerra, IEEE Member, Juan Aguirre, Juan E. Ortuño, Maria J. Ledesma-Carbayo, IEEE Member, Juan J. \\ Vaquero, IEEE Senior Member, Manuel Desco and Andres Santos, IEEE SeniorMember
}

\begin{abstract}
Monte Carlo methods provide a flexible and rigorous solution to the problem of light transport in turbid media, which enable approaching complex geometries for a closed analytical solution is not feasible. The simulator implements local rules of propagation in the form of probability density functions that depend on the local optical properties of the tissue.

This work presents a flexible simulator that can be applied in multiple applications related to optical tomography. In particular, unlike previous codes, the simulator explicitly supports fluorescent-tissues and variance reduction and code parallelization techniques are implemented in order to speed up the execution with fluorochrome-labelled agents.

The simulator is validated with simple geometries for which an analytical solution exists, as well as with an experimental polyester resin based optical phantom.
\end{abstract}

\section{INTRODUCTION}

$\mathrm{T}^{\mathrm{N}}$ HE complicated nature of photon propagation in tissue makes the exact solution of the Maxwell's equations a very difficult endeavor. The radiative transfer equation (RTE), a differential equation describing radiance that is derived from energy conservation principles, is widely accepted as an accurate model for light propagation in tissues. [1]. However, the RTE does not have closed analytical solutions for arbitrary geometries and finite element methods (FEM) numerical solutions with sufficiently dense discretizations lead to computationally expensive problems. The RTE can also be solved with a Monte Carlo (MC) simulation by modeling local rules of propagation and tracking the fate of thousands of independent photons.

This work deals with photon transport simulation in a complex setup in which multiple light sources illuminates the sample and several detectors are placed around the object. The goal is to develope a flexible code capable of simulating diagnostic [2, 3] and therapeutic [4] applications, with

Manuscript received November 2, 2009. This work was supported in part by the Ministry of Science and Innovation under projects TEC2008-06715 and TEC2007-64731/TCM and by the EU's $7^{\text {th }}$ Frame Programme under contract HEALTH-F5-2008-201792

P. Guerra and J. Ortuño are with the Research Centre for Bioengineering, Biomaterials and Nanomedicine, 58018 Zaragoza, Spain (email: pguerra@ciber-bbn.es)

J. Aguirre, Juan J. Vaquero and M. Desco are with the Unidad de Medicina Cirugia Experimental, Hospital General Universitario Gregorio Marañon, 28007 Madrid, Spain.

A. Santos and M.J. Ledesma are with Electronic Engineering Dpt., Universidad Politécnica de Madrid, 28040 Madrid, Spain. particular emphasis on optical tomography, either diffuse (DFOT) [5, 6], or projective [7]. Fluorescence optical tomography (FOT), is a particular case of DFOT, where the aim is to retrieve the localization of fluorochrome-labeled agents. The presence of these molecules, leads to revisiting existing codes, such as MCML [8], in order to incorporate the physical process of light absorption by the fluorochromes and later reemission at a longer wavelength.

The document presents the physical model that has been considered, outlines the execution kernel of the simulator and present some validation results.

\section{MATERIAL AND METHODS}

\section{A. Physics Model}

\section{1) Photon Description}

Photons are described by the following variables: weight $(w)$, the current spatial coordinates $(X)$, time $(\mathrm{t})$, its current wavelength $(\lambda)$, the photon local axes coordinates $C^{\prime}=\left(i^{\prime}, j^{\prime}, k^{\prime}\right)$, being $\mathrm{k}^{\prime}$ also the propagation vector $U$, and current location within the voxellized tissue. Cartesian coordinates are preferred because in this case formulas for propagation are simpler [9], and the angle variables describing photon direction do not change unless the photon's direction changes.

\section{2) Tissue Model}

The object is discretized into voxels, each of which is represented by tissue type and fluorescent properties, resulting the tuple $\left(\mu_{a}, \mu_{s}, g, n, \mu_{a}, \boldsymbol{\Phi}\right)$, that correspond with the absorption coefficient $\left(\mathrm{cm}^{-1}\right)$, the scattering coefficient $\left(\mathrm{cm}^{-1}\right)$, the anisotropy factor, the index of refraction, the fluorophore absorption coefficient $\left(\mathrm{cm}^{-1}\right)$ and the fluorophore quantum yield respectively. Biological tissues in the near infrared (NIR) range are strongly forward scattering with anisotropy factors typically in the range $0.69<\mathrm{g}<0.99$.

3) Fluorochrome Model

A fluorophore is a component of a molecule which causes a molecule to be fluorescent: absorbs photons at wavelength $\lambda_{i}$ and re-emits them at a longer wavelength $\lambda_{\mathrm{f}}$. The emission wavelength $\lambda_{\mathrm{f}}$ is independent of the excitation wavelength; however the efficiency of this energy transfer, quantum efficiency $\phi$, does depend on $\lambda_{\mathrm{i}}$. Moreover, the time required for the molecule to relax and return to ground state, thus emitting a photon at $\lambda_{\mathrm{i}}$, is characteristic of each fluorochrome. 
There is no limitation in the number of fluorescent agents than can be attached to tissue types; thus enabling different concentrations and types of fluorescent-dyes. There is also no constraint in the absorption and emission spectrum of the fluorochromes, though current simulations assume a constant yield and a monochromatic reemission.

In case of using different dyes, crosstalk effects are by construction part of the simulation.

\section{4) Photon-matter interaction}

At each interaction point $\mathrm{C}$, a photon $p$ may either interact with the tissue or a fluorophore. In the former case the photon is either absorbed or scattered while in the later case the photon is either absorbed by the fluorophore or reemitted at a longer wavelength. Tissue and fluorophore properties may be lumped together [10] to define an equivalent attenuation coefficient $\mu_{a c}$ and yield $\Phi_{a c}$ :

$$
\begin{aligned}
& \mu_{a c}=\mu_{a}+\mu_{a f} \\
& \mu_{s c}=\mu_{a} \\
& \Phi_{c}=\frac{\mu_{a f}}{\mu_{a}+\mu_{a f}} \cdot \Phi
\end{aligned}
$$

These definitions are convenient for obtaining the probably of each of the possible outcomes of the photon-matter interaction: scattering $\left(p_{s}\right)$, absorption in tissue or fluorochrome $\left(p_{a}\right)$ and fluorochrome absorption-remission $\left(p_{e f}\right)$, which are given by:

$$
\begin{aligned}
& p_{s}=\frac{\mu_{s}}{\mu_{a c}+\mu_{s}} \\
& p_{a}=\frac{\mu_{a c}}{\mu_{a c}+\mu_{s}} \cdot\left(1-\Phi_{c}\right) \\
& p_{e f}=\frac{\mu_{a c}}{\mu_{a c}+\mu_{s}} \cdot \Phi_{c}
\end{aligned}
$$

In an ideal MC implementation, a coin would be tossed to determine the photon fate and in case of absorption, photon dies and a new photon is taken from the laser. This work considers forced interactions in order to reduce variances. This approach implies that at each interaction the photon packet is split into three fractions, each of which undergoes on of the possible processes. From a practical perspective, the consequence of this approach is that at tissue voxels labeled with fluorochromes, the initial photon packet splits in two: one that represents the fraction of photons that undergo scattering and the other the fraction of absorbed photons that are reemitted by the fluorophore. The simulator kernel handles this situation by placing the reemitted photon in a queue for later simulation and continuing the simulation with the scattered photon.

Whenever a photon is absorbed by the tissue and reemitted at a different wavelength, tissue property tables are updated accordingly, this procedure overcomes the limitations of other simulation codes [9].

\section{5) Photon scatering}

The differential scattering cross section is referred to as the phase function $p(\theta, \varphi)$, which specifies the probability of scattering into a unit solid angle $(\theta, \varphi)$, measured from the incident direction. The average over the solid angle of $p(\theta, \varphi)$ is known as the asymmetry parameter, which ranges from backward ( $\mathrm{g}=-1)$ to forward scattering $(\mathrm{g}=1)$. A photon is considered to acquired random direction after approximately $1 /(1-g)$ scatters. The Henyey-Greenstein phase function [11] is used to characterize the angular distribution of scattered light by tissue. This function is parameterized by the average cosine of the scattering angle $<\cos (\theta)>$, i.e. the anisotropy factor $g$ :

$$
p_{H G}(\cos \theta)=\frac{1}{2} \cdot \frac{1-g^{2}}{\left(1+g^{2}-2 \cdot g \cdot \cos \theta\right)^{\frac{3}{2}}}
$$

\section{6) Photon mechanics}

Photon mechanics involves translations due to propagation as well rotations due to scattering, reflection or refraction at tissue interfaces. Photon local axes are defined such that $\boldsymbol{B}_{\boldsymbol{c}}$ represents the matrix transformation between the local reference system $\mathbf{C}^{\prime}$ and the world reference system $\mathbf{C}$ and the propagation vector in the world system $U$ aligns with the k'axis in the local reference frame. That is,

$$
\begin{aligned}
& U_{L}=(0,0,1)^{T} \\
& U=B_{c} \cdot U_{L}
\end{aligned}
$$

With this definition, for a photon located at $X=(x, y, z)$ traveling a distance $\Delta s$ in the direction $U=\left(u_{x}, u_{y}, u_{z}\right)$, the new coordinates $\mathrm{X}^{\prime}=\left(\mathrm{x}^{\prime}, \mathrm{y}^{\prime}, \mathrm{z}^{\prime}\right)$ are given by:

$$
X^{(i+1}=X^{(i}+\Delta s \cdot B_{c} \cdot U_{L}
$$

Reflections and refraction take place at the interface between two tissues; this interface is defined by the normal vector $n$. In both cases, the new direction of propagation $U^{\prime}$ belongs to the plane defined by $\boldsymbol{U}$ and $n$ and it is computed as a rotation around the vector normal to the plane $S=U x n$. Rotations are implemented on the transformed system $C^{\prime \prime}$ as a rotation $\boldsymbol{R}$ around $S$, as:

$B_{c}^{(i+1}=B_{r} \cdot R \cdot B_{r}^{T} B_{c}^{(i}$

Where the rotation matrix $\mathbf{B}_{\mathbf{r}}$ is defined as $\left((n x S)^{T}, n, S^{T}\right)$. In the case of reflection the rotation angle will be $\left(p-2 \alpha_{V}\right)$ while in the case of refraction it will be $\left(a_{V^{\prime}} a_{V}\right)$.

Photon-matter scattering inside the voxel is represented as deflection angle $\theta$ and a azimuth angle $\varphi$, with respect to the propagation direction $\boldsymbol{U}=\left(u_{x}, u_{y}, u_{z}\right)$. This rotation transforms the photont reference vectors: 


$$
\begin{aligned}
& B_{d}=\left(\begin{array}{ccc}
\frac{u_{x} \cdot u_{z}}{s_{z}} & -\frac{u_{y}}{s_{z}} & u_{x} \\
\frac{u_{y} \cdot u_{z}}{s_{z}} & \frac{u_{x}}{s_{z}} & u_{y} \\
-s_{z} & 0 & u_{z}
\end{array}\right) \cdot\left(\begin{array}{c}
\cos \Phi \sin \theta \\
\sin \Phi \sin \theta \\
\cos \theta
\end{array}\right) \\
& s_{z}=\sqrt{1-u_{z}^{2}} \\
& B_{c}^{(i+1}=B_{d} \cdot B_{c}^{(i}
\end{aligned}
$$

\section{7) Physical Data Collection}

During the simulation several physical parameters are recorded, for both injected photons at wavelength $\lambda_{\mathrm{i}}$ as well as the reemitted photons at wavelength $\lambda_{\mathrm{F}}$ are measured, including reflectance, transmittance and fluence. Photon packet weights are normalized to the laser wavelength $\lambda_{\mathrm{i}}$ in order to represent energy.

At each interaction point the absorbed energy is stored in a matrix $\mathbf{A}_{\mathbf{x y z}}$, which represents the total deposited energy at each voxel and it is proportional to the photon fluence $\Phi(\mathrm{r}, \lambda)$. Fluence is computed out of the absorbed energy, adjusting for a scale parameter in order to fulfill that the exiting photon flux, consisting of total transmittance $T_{t}$ and total radiance $T_{r}$, plus the absorbed energy at different wavelengths, $\Phi(\mathrm{r}, \lambda) \mu(\mathrm{r}, \lambda)$ equals the injected energy $\mathrm{E}$ [12]:

$$
T_{t}+T_{r}+\sum_{\lambda} \sum_{\text {Volumen }} \Phi_{x y z}(r, \lambda) \cdot \mu_{a}(r, \lambda) \cdot V_{\text {voxel }}=E
$$

\section{B. MC Simulator kernel}

\section{1) Tools}

The simulator has been coded in $\mathrm{C}++$, threaded for multiprocessor environments with OpenMP 3.0 [13] and compiled with Intel Compiler 11.0 (Intel Corp., 2200 Mission College Blvd Santa Clara, CA, USA).

\section{2) Kernel Core}

The simulator follows an execution flow similar to that describe by Wang [8], with the added feature that tissues may be labeled with fluorochromes, something that was not possible with previous codes [9]. Additionally the simulator is fully extended to 3D and tracks photon timing and rotations enabling intensity modulated simulations and polarization considerations. For each photon packet, the following steps are carried out:

1. Select the photon for simulation, which may come from the queue previously mentioned or from the laser.

2. Update tissue tables to reflect properties at the current wavelength.
3. Compute the distance before the interaction point and translate the photon. Displacement may be such that more than one voxel is crossed. This requires updating the actual distances to reflect variations in tissue properties.

4. Compute the photon fraction that undergoes absorption, scattering and reemission. Store the reemission factor in the queue if its weight is higher than 0 .

5. Apply Russian roulette to the photon: if its weight is lower than a given threshold a coin is tossed to determine whether the photon is absorbed or its weight is increased.

6. Iterate through steps 3-5 until the photon escapes the voxellized volume or it is completely absorbed.

7. Check whether the photon impinges on the external detectors.

\section{RESULTS}

\section{A. Simulator validation against analytical solutions}

The diffusion approximation (DA) equation [9] and data from existing literature is used to validate the code with simple slab geometries [14, 15]. Firstly, estimated observable physical values of the total reflectance and transmittance provided by the simulator is checked against published literature. The match of the results, summarized in the next tables, confirms the correctness of data scale factors and photon weightings.

TABLE 1: REFLECTANCE AND TRANSMITTANCE FOR A SEMI-INFINITE BLOCK

\begin{tabular}{|l|c|c|c|c|}
\hline Author & $R$ & R error & $T$ & T error \\
\hline Giovanelli [16] & 0.26000 & - & 0 & 0 \\
\hline Prahl . [17] & 0.26079 & 0.00079 & 0 & 0 \\
\hline Wang et al [8] & 0.25907 & 0.00170 & 0 & 0 \\
\hline This work & 0.25936 & 0.00142 & 0 & 0 \\
\hline
\end{tabular}

TABLE 2: REFLECTANCE AND TRANSMITTANCE FOR A SLAB BLOCK

\begin{tabular}{|l|l|l|l|l|}
\hline Author & \multicolumn{1}{|c|}{$R$} & R error & $T$ & T error \\
\hline van de Hulst [18] & 0.09739 & - & 0.66096 & \\
\hline Prahl [17] & 0.09711 & 0.00033 & 0.66159 & 0.00049 \\
\hline Wang et al [8] & 0.09734 & 0.00033 & 0.66096 & 0.00020 \\
\hline This work & 0.0969 & $9.43 \times 10^{-4}$ & 0.66160 & 0.00120 \\
\hline
\end{tabular}

Next, the estimated photon fluence $\Phi(r, \lambda)$ is compared with the value provided by the DA for a slab geometry of thickness $\mathrm{L}=4 \mathrm{~cm}$ when taking into account the attenuation profile of the tissue [19].

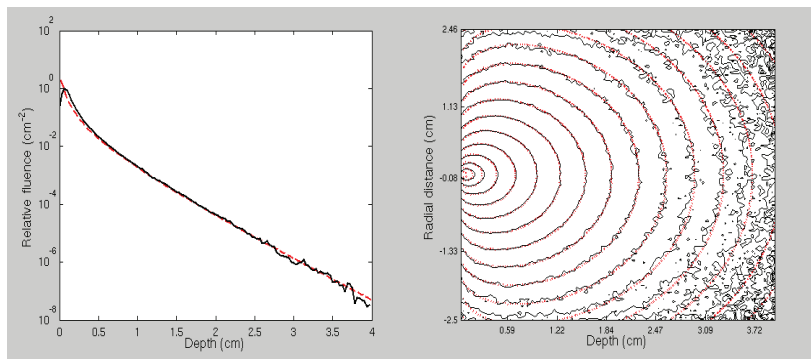

Figure 1: Longitudinal profile and axial cut of the fluence computed with the MC simulator and with the DA.

Figure 2 shows the fluence profile and a slice cut in logarithmic scale along the laser axis for the MC simulation 
(solid line), with $5 * 10^{7}$ photons, and the DA analytical solution (dotted line) for a $4 \mathrm{~cm}$ slab geometry with the following optical parameters: $\mu_{a}=0.3 \mathrm{~cm}-1, \mu_{s}=100 \mathrm{~cm}^{-1}$, $g=0.9, D=0.033 \mathrm{~cm}$. As expected, except in points close to the surface where the laser impinges the tissue, there is complete correspondence between both solutions

\section{B. Simulator validation against experimental data}

The Monte Carlo simulator is validated against real measurements with an optical phantom. The scattering coefficient and the absorption coefficient of the phantom can be tuned according to the concentration of absorber and scatterers [20]. For the studies presented herein we made several phantoms using polyester resin as bulk matrix, Pro-Jet ink $900 \mathrm{NP}$ (ICI plc., Slough, UK) as absorber and $\mathrm{TiO}_{2}$ as scatterer [20] whose optical parameters are $\left(\mu_{a}, \mu_{s}, g\right)=(0.1 \mathrm{~cm}$ $\left.{ }^{1}, 8 \mathrm{~cm}^{-1}, 0.9\right)$.
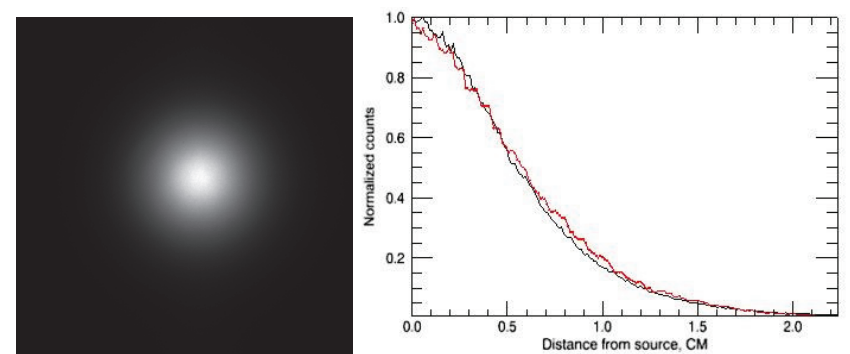

Figure 2: Experimental laser spot on the detector (left) and radial profile of the experimental spot and the MC simulation output when illuminating the resin phantom.

\section{CONCLUSIONS AND Future Work}

This work has presented a MC simulator for the transport of light in biological tissue that explicitly supports fluorescent activity inside the tissue. This feature enables the simulation of optical tomographs with complex structures, such as small animal phantoms and will provide a way to better understand photon propagation issues and thus optimize reconstruction algorithms. Moreover, the integration of fluorescence in the simulator enables the simulation of second-order effects, such as crosstalk between dyes and variations of optical tissue properties with wave length, that are usually neglected by analytical solutions.

Further validation with fluorochrome sources is in progress as well as an assessment of actual speed up achieved with the implemented multiprocessor threading.

\section{REFERENCES}

[1] O. Dorn, "A transport-backtransport method for optical tomography," Inv. Probl., vol. 14, no. 5, pp. 1107-1130, 1998. S. K. Chang, D. Arifler, R. Drezek et al., "Analytical model to describe fluorescence spectra of normal and preneoplastic epithelial tissue: comparison with Monte Carlo simulations and clinical measurements," Journal of Biomedical Optics, vol. 9, no. 3, pp. 511, 2004.

[3] I. Pavlova, C. R. Weber, R. A. Schwarz et al., "Monte Carlo model to describe depth selective fluorescence spectra of epithelial tissue: applications for diagnosis of oral precancer," Journal of Biomedical Optics, vol. 13, no. 6, pp. 064012, 2008.

[4] L. H. P. Murrer, J. P. A. Marijnissen, and W. M. Star, "Ex vivo light dosimetry and Monte Carlo simulations for endobronchial photodynamic therapy," Physics in Medicine and Biology, vol. 40, no. 11, pp. 1807, 1995.

[5] D. A. Boas, D. H. Brooks, E. L. Miller et al., "Imaging the body with diffuse optical tomography," Signal Processing Magazine, IEEE, vol. 18, no. 6, pp. 57, 2001.

[6] R. B. Schulz, J. Ripoll, and V. Ntziachristos, "Noncontact optical tomography of turbid media," Opt. Lett., vol. 28, no. 18, pp. 1701, 2003.

[7] M. A. Haidekker, "Optical transillumination tomography with tolerance against refraction mismatch," Computer Methods and Programs in Biomedicine, vol. 80, no. 3, pp. 225, 2005.

[8] L. Wang, S. L. Jacques, and L. Zheng, "MCML--Monte Carlo modeling of light transport in multi-layered tissues," Computer Methods and Programs in Biomedicine, vol. 47, no. 2, pp. 131, 1995.

[9] T. J. Pfefer, J. Kehlet Barton, E. K. Chan et al., "A three-dimensional modular adaptable grid numerical model for light propagation during laser irradiation of skin tissue," Selected Topics in Quantum Electronics, IEEE Journal of, vol. 2, no. 4, pp. 934, 1996.

[10] Q. Liu, C. Zhu, and N. Ramanujam, "Experimental validation of Monte Carlo modeling of fluorescence in tissues in the UV-visible spectrum," Journal of Biomedical Optics, vol. 8, no. 2, pp. 223-236, 2003.

[11] L. G. Henyey, and J. L. Greenstein, "Diffuse radiation in the galaxy," Annales d'Astrophysique, vol. 93, pp. 70, 1941.

[12] D. A. Boas, J. P. Culver, J. J. Stott et al., "Three dimensional Monte Carlo code for photon migration through complex heterogeneous media including the adult human head," Optics Express, vol. 10, no. 3, pp. 159, 2002.

[13] OpenMP Architecture Review Board, OpenMP Application Program Interface http://openmp.org, 2008.

[14] R.C. Haskell, L.O. Svaasand, T.T. Tsay et al., "Boundary-Conditions For Diffusion Equation in Radiative- Transfer," J. Opt. Soc. Am. A Optics Image Science and Vision, vol. 11, pp. 2727-2741, 1994.

[15] M. S. Patterson, B. Chance, and B. C. Wilson, "Time resolved reflectance and transmittance for the non-invasive measument of tissue optical properties," Applied Optics, vol. 28 (12), pp. 23312336, 1989.

[16] R. G. Giovanelli, "Reflection by Semi-infinite Diffusers," Journal of Modern Optics, vol. 2(4), pp. 153, 1955.

[17] S. A. Prahl, M. Keijzer, S. L. Jacques et al., "A Monte Carlo Model of Light Propagation in Tissue," SPIE Institute Series, vol. 5, pp. 102,1989

[18] H. C. van de Hulst, Light Scattering by Small Particles: Dover, 1981.

[19] A. Garofalakis, G. Zacharakis, G. Filippidis et al., "Characterization of the reduced scattering coefficient for optically thin samples: theory and experiments," Journal of Optics A: Pure and Applied Optics, vol. 6, pp. 725, 2004.

[20] B. W. Pogue, and M. S. Patterson, "Review of tissue simulating phantoms for optical spectroscopy, imaging and dosimetry," Journal of Biomedical Optics, vol. 11, no. 4, pp. 041102, 2006. 